\title{
Suicide By Asphyxiation Due to Helium Inhalation
}

\author{
Matthew O. Howard, PhD, * Martin T. Hall, PhD, † Jeffrey D. Edwards, MSW, * Michael G. Vaughn, PhD, $f$ \\ Brian E. Perron, PhD, $\S$ and Ruth E. Winecker, PhD
}

\begin{abstract}
Suicide by asphyxiation using helium is the most widely promoted method of "self-deliverance" by right-to-die advocates. However, little is known about persons committing such suicides or the circumstances and manner in which they are completed. Prior reports of suicides by asphyxiation involving helium were reviewed and deaths determined by the North Carolina Office of the Chief Medical Examiner to be helium-associated asphyxial suicides occurring between January 1, 2000 and December 31, 2008 were included in a new case series examined in this article. The 10 asphyxial suicides involving helium identified in North Carolina tended to occur almost exclusively in non-Hispanic, white men who were relatively young $(\mathrm{M}$ age $=41.1 \pm 11.6)$. In 6 of 10 cases, decedents suffered from significant psychiatric dysfunction; in 3 of these 6 cases, psychiatric disorders were present comorbidly with substance abuse. In none these cases were decedents suffering from terminal illness. Most persons committing suicide with helium were free of terminal illness but suffered from psychiatric and/or substance use disorders.
\end{abstract}

Key Words: asphyxia, helium, suicide, right-to-life

(Am J Forensic Med Pathol 2010;XX: 000-000)

$\mathrm{P}$ ublication, in 1991, of the right-to-die manifesto and suicide "how-to" guide, Final Exit: The Practicalities of Self-Deliverance and Assisted Suicide for the Dying, ${ }^{1}$ raised a maelstrom of controversy regarding the appropriateness of suicide as a response to terminal or "hopeless" physical illness and exposed divisions within the right-to-die movement itself. In the 1990 s, many right-to-die advocates were engaged in public education as to the purported virtues of advanced directives, living wills, and legalized physicianassisted suicide. ${ }^{2}$ At the same time, other elements of this movement, including the Self-Deliverance New Technology (NuTech) Group, were developing technologies to "empower people to die on their own terms by controlling the timing and manner of their own death."2 (p. 8) NuTech members, including Derek Humphry, author of Final Exit, sought to identify multiple suicide methods that were swift, painless, failure-proof, inexpensive, and nondisfiguring. The group also considered it vital that the method be simple, leave little or no indication that the death was unnatural in nature, and not require a physician's assistance or prescription. ${ }^{2}$

With its detailed descriptions of diverse suicide methods and specific endorsement of the plastic bag asphyxiation method, publication of Final Exit brought an easily understood and generally

Manuscript received January 19, 2010; accepted March 3, 2010.

From the ${ }^{*}$ School of Social Work, University of North Carolina at Chapel Hill; †Department of Behavioral Medicine, School of Medicine, University of Kentucky; $\$$ School of Public Health, Saint Louis University; §Department of Psychiatry, School of Medicine, University of Michigan; and $\mathbb{\| N o r t h}$ Carolina Office of the Chief Medical Examiner.

Supported by NIH grants DA15929, DA15556, DA021405 (M.O.H.) and DA007304 (M.T.H.).

Correspondence: Matthew O. Howard, Frank Daniels Distinguished Professor, Tate-Turner-Kuralt Building, 325 Pittsboro, CB 3550, Chapel Hill, NC 27599-3550. E-mail: mohoward@email.unc.edu.

Copyright (C) 2010 by Lippincott Williams \& Wilkins

ISSN: 0195-7910/10/0000-0001

DOI: $10.1097 /$ PAF.0b013e3181ed7a2d effective suicide method to the masses. The book was a commercial success, appearing on the New York Times bestseller list and selling more than 1.5 million copies in the decade following its publication. In 2007, Final Exit was named one of the 25 most influential books of the past quarter-century by book critics and editors of USA Today. ${ }^{3}$

Concerns that suicides in nonterminally ill depressed persons might follow exposure to methods elucidated in Final Exit were soon raised, ${ }^{4}$ and dramatic increases in plastic bag asphyxial suicides were observed in New York City ${ }^{5}$ and the United States 6 in the year following publication of Final Exit. Investigators concluded that "most persons exposed to Final Exit were not terminally ill and had used it as a suicide manual ... (and that) it is likely that a psychiatric disorder would have been diagnosed in most of these people."5 (p. 1509)

Efforts by NuTech and others to develop a more effective suicide method and widely disseminate it to the public have continued to the present. In 2000, a supplement to Final Exit was published that presented the first description of helium-assisted plastic bag asphyxiation. ${ }^{7}$ Advocates emphasized the enhanced lethality of this approach, reduction in time required for death to occur to less than 5 minutes, and elimination of the need for a sedative prescription. Proponents of the method also noted that materials needed to complete such suicides are readily accessible and that asphyxiation due to helium inhalation is often undetected by autopsy (where findings are typically nonspecific) or toxicological analysis (because special sampling and assay methods are required). Thus, such suicides are likely to remain undetected in cases where the helium delivery apparatus and plastic bag are removed before the death scene is examined and no other information is available implicating death by helium-assisted asphyxiation. Modifications of the helium method were published in $2002^{8}$ and $2009,{ }^{9}$ a DVD including a step-by-step demonstration of the method is available for purchase, ${ }^{10}$ and instructional videos depicting the method are accessible on the internet. A schematic of the helium delivery apparatus is presented in Figure 1. ${ }^{9}$

Given the recent development, broad dissemination, and notable lethality of helium-assisted suicide, we endeavored to better understand characteristics of suicides by this method. First, we reviewed findings of extant studies examining suicides by asphyxiation due to helium inhalation. Second, we report new findings from the largest series of these suicides heretofore examined. Results of this investigation may lead to improved identification of heliumassisted suicides by medical examiners, enhanced screening and prevention efforts on the part of physicians and other professionals treating individuals at risk for suicide, and shed new light on unintended deleterious consequences of widespread dissemination of detailed suicide methods to the general public.

\section{MATERIALS AND METHODS}

The current report presents findings from 2 related studies. The first is a review of published investigations of suicides by asphyxiation due to helium inhalation. The second is a case series of suicides by asphyxiation due to helium inhalation occurring in North Carolina between 2000 (the year in which the method was first described) and December 31, 2008. 


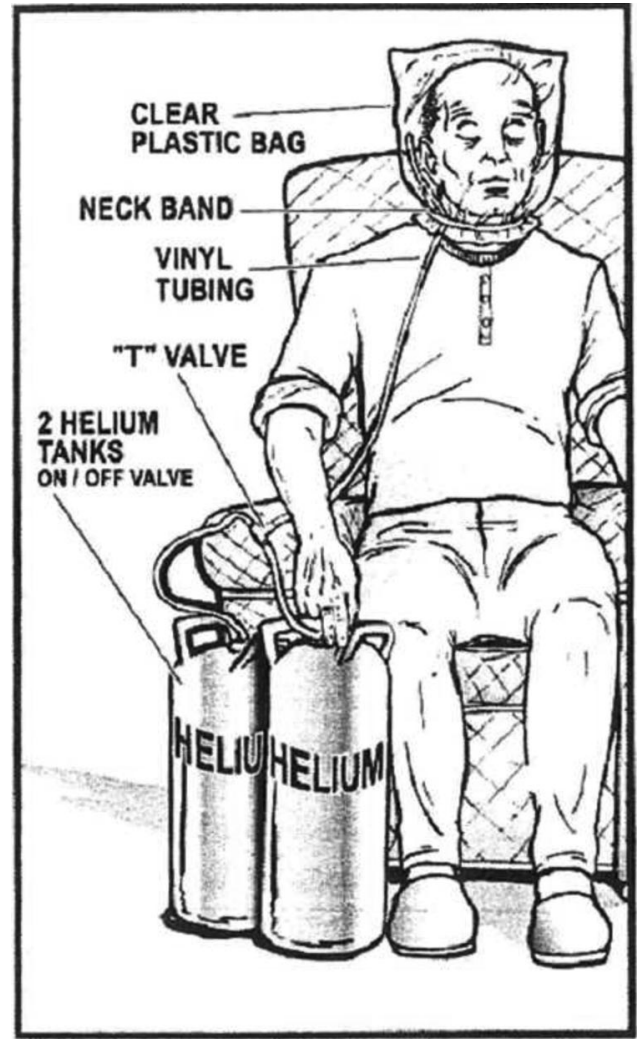

FIGURE 1. Schematic of plastic bag asphyxiation suicide using helium gas in final exit. Reprinted with permission from Final Exit: The Practicalities of Self-deliverance and Assisted Suicide for the Dying. ${ }^{9}$ (p.4)

\section{Identification of Published Reports}

A broad search of the general medical literature was undertaken for any relevant reports addressing suicide by asphyxiation due to helium inhalation. This process entailed searching the PubMed database for the period January 1, 1957 to November 1, 2009 using the search phrase "suicide and helium." Seven pertinent records were identified as follows: 6 English-language case studies $^{11-16}$ and a Danish-language case study. ${ }^{17}$ A search of EMBASE using the identical approach for the period January 1, 1988 to November 1, 2009 identified the same 7 reports. The 6 Englishlanguage reports relevant to this review were published between 2002 and 2007 and present a total of 14 cases. ${ }^{1-16}$ The Danish study included a synoptic abstract in English indicating that the decedent was a 35-year-old man who had committed suicide with a plastic bag and helium using a "new and highly lethal technique." $" 17$ The case reports included in this review constitute the entirety of published research on helium-assisted suicide and are presented in Table 1.

\section{Identification of Suicides by Asphyxiation Due to Helium Inhalation in North Carolina}

All deaths determined by the North Carolina Office of the Chief Medical Examiner (NCOCME) to be asphyxial suicides due to helium inhalation that occurred between January 1, 2000 and December 31, 2008, were included in this study. These suicides were identified through a search of the manner and cause of death fields of the electronic records maintained by the NCOCME. The presence of helium was confirmed by toxicological testing in 9 of 10 identi- fied cases. Only the first reported case (ie, 2001) was not subjected to toxicological testing for helium. Specimens from suspected helium asphyxiation cases autopsied at the NCOCME are collected in $20 \mathrm{~mL}$ headspace vials. In some cases, given that one central laboratory conducts testing for all medical examiner cases in the state, blood samples are delivered to the NCOCME in standard collection vials. Immediately upon arrival, $5 \mathrm{~mL}$ of blood from the standard autopsy vial is transferred to a headspace vial for later analysis. Medical records associated with these deaths were manually reviewed and abstracted including the OCME Report of Investigation, State of North Carolina Death Certificate, Report of Autopsy, Toxicology Report, Case Encounter Form, Pathologist's Notes, and Supplemental Report of Cause of Death. On January 5, 2010, the University of North Carolina Institutional Review Board determined that the reported research does not require Institutional Review Board approval under pertinent federal regulations. Characteristics of the 10 cases identified are presented in Table 2.

\section{RESULTS}

\section{Review of Published Cases}

The first death attributed to suicide by asphyxiation due to helium inhalation reported in the medical literature occurred in September $2000,{ }^{11}$ shortly after the description of the method was published. Several investigators asserted that suicides by the helium method had not been seen in their localities prior to publication of the 2000 Supplement to Final Exit. ${ }^{11,12,15,16}$

The 14 decedents whose cases were presented in the 6 published reports ranged in age from 19 to 81 ( $\mathrm{M}$ age $=50.0, \mathrm{SD}=$ 21.8 , median $=48.5)$. Between these extremes, decedents were approximately evenly divided between those in their $20 \mathrm{~s}, 30 \mathrm{~s}, 40 \mathrm{~s}$, $60 \mathrm{~s}$, and 70s. Medical and psychiatric histories were scant or entirely unreported for some cases, but revealed a history of depression, prior suicide attempt(s), paranoid schizophrenia, or some combination thereof in $4(25.6 \%)$ cases. In $4(25.6 \%)$ additional cases, psychiatric dysfunction may have contributed to the suicide, given that 3 of these decedents were determined to be in good health (ages 49, 49, and 76) and one mentioned the recent death of his wife as a reason for his suicide in a note left at the death scene. In 5 other cases (including 4 decedents in their 20 s or 30 s), no medical or psychiatric histories were reported. A terminal disease process was present in only 2 of $14(14.3 \%)$ cases. In $2(14.3 \%)$ additional cases involving men ages 71 and 78, "failing health" and "unspecified health problems" were possible contributing factors. Medical disorders were not implicated in 10 of $14(71.4 \%)$ suicides.

In all reported cases, routine toxicological testing did not reveal the presence of helium and manner and cause of death determinations relied heavily on death scene investigations. Autopsy findings tended to be absent or nonspecific in the 12 cases that involved an autopsy.

In 8 cases $(57.1 \%)$, a suicide note was found, and in 4 cases (28.6\%) right-to-die literature was found at the death scene.

A number of helium delivery devices were employed. Five cases involved use of a mask; 4 of these cases were reported in 2002 or 2003, before plastic bag asphyxiation (without use of a mask) became preferred by advocates of the helium method. ${ }^{8}$ Characteristics of the plastic tubing used, use of rubber bands and Velcro straps to secure plastic bags to the neck, types of helium canisters employed, and use of multiple plastic bags in 1 case were consistent with published descriptions of helium-assisted suicide. ${ }^{8}$

\section{Characteristics of Suicides by Asphyxiation due to Helium Inhalation in North Carolina}

Asphyxial suicides in North Carolina involving helium inhalation tended to occur almost exclusively in non-Hispanic, white 


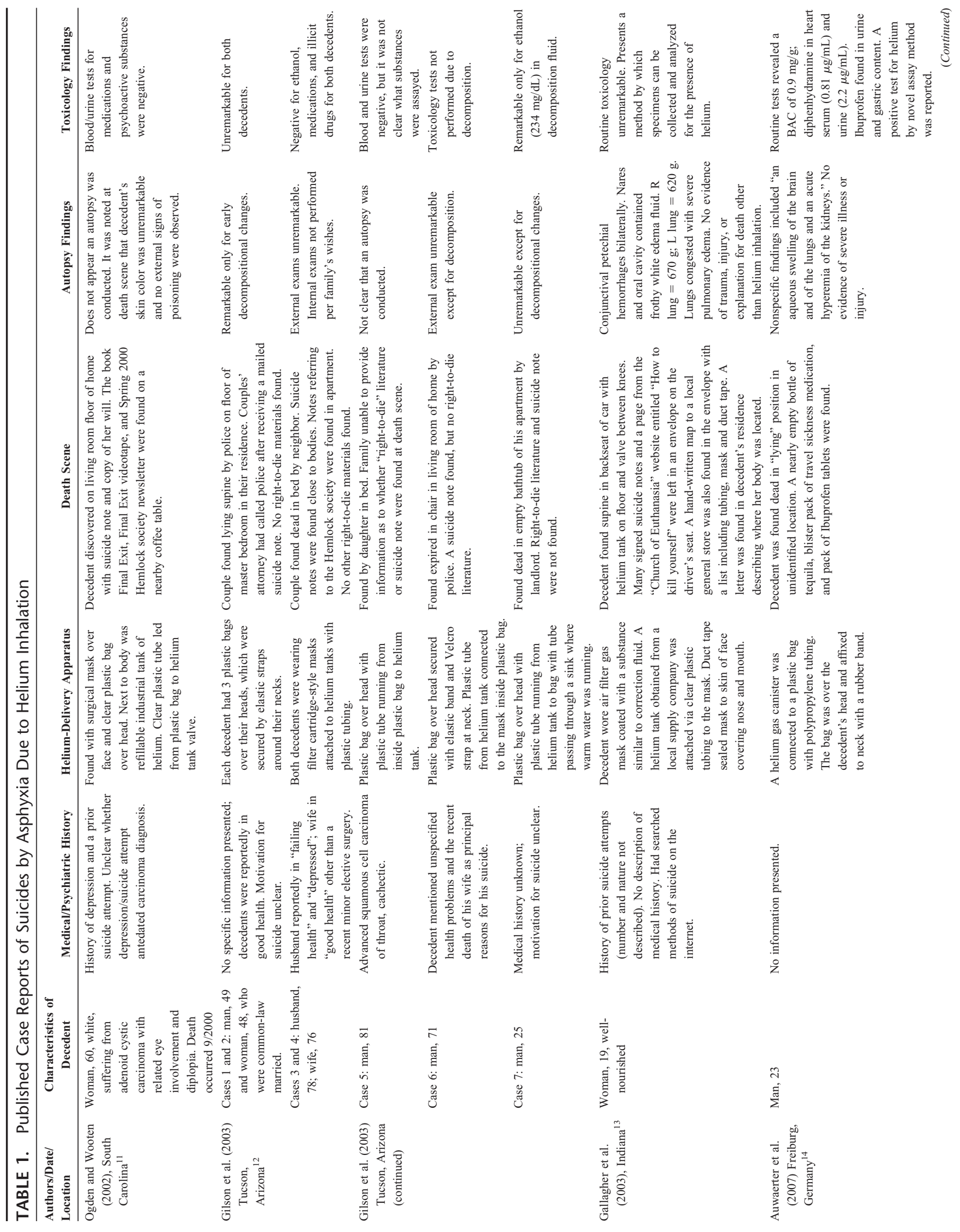




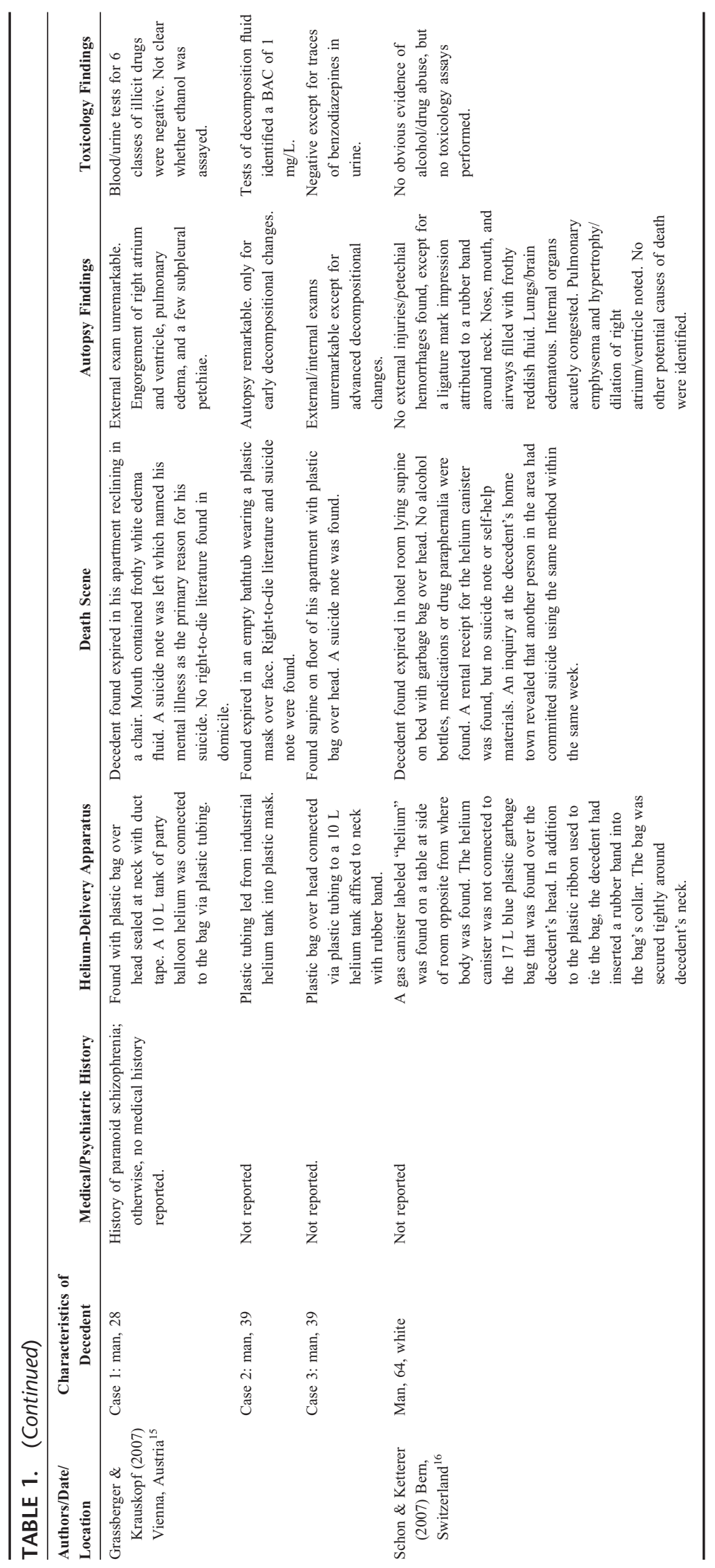




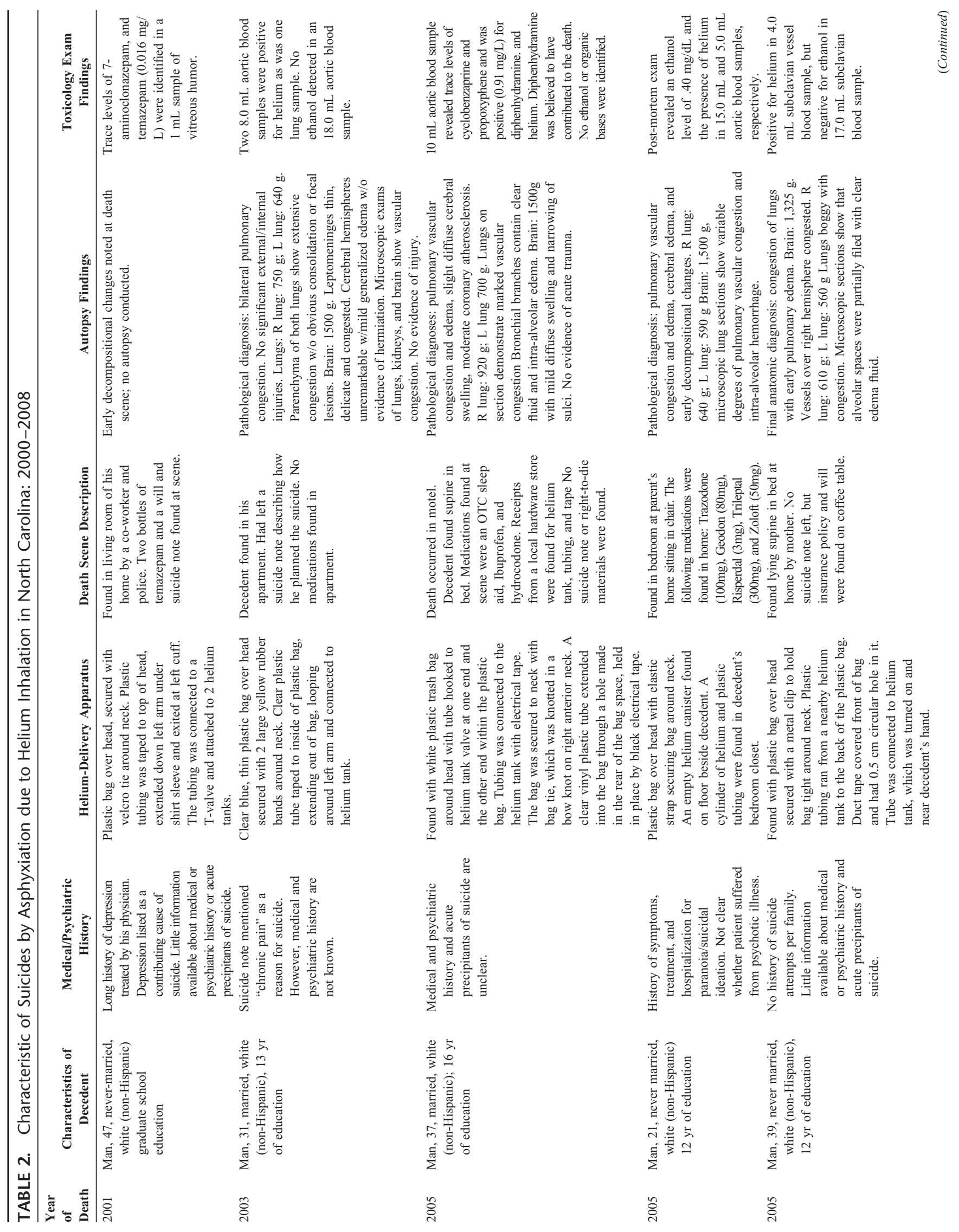




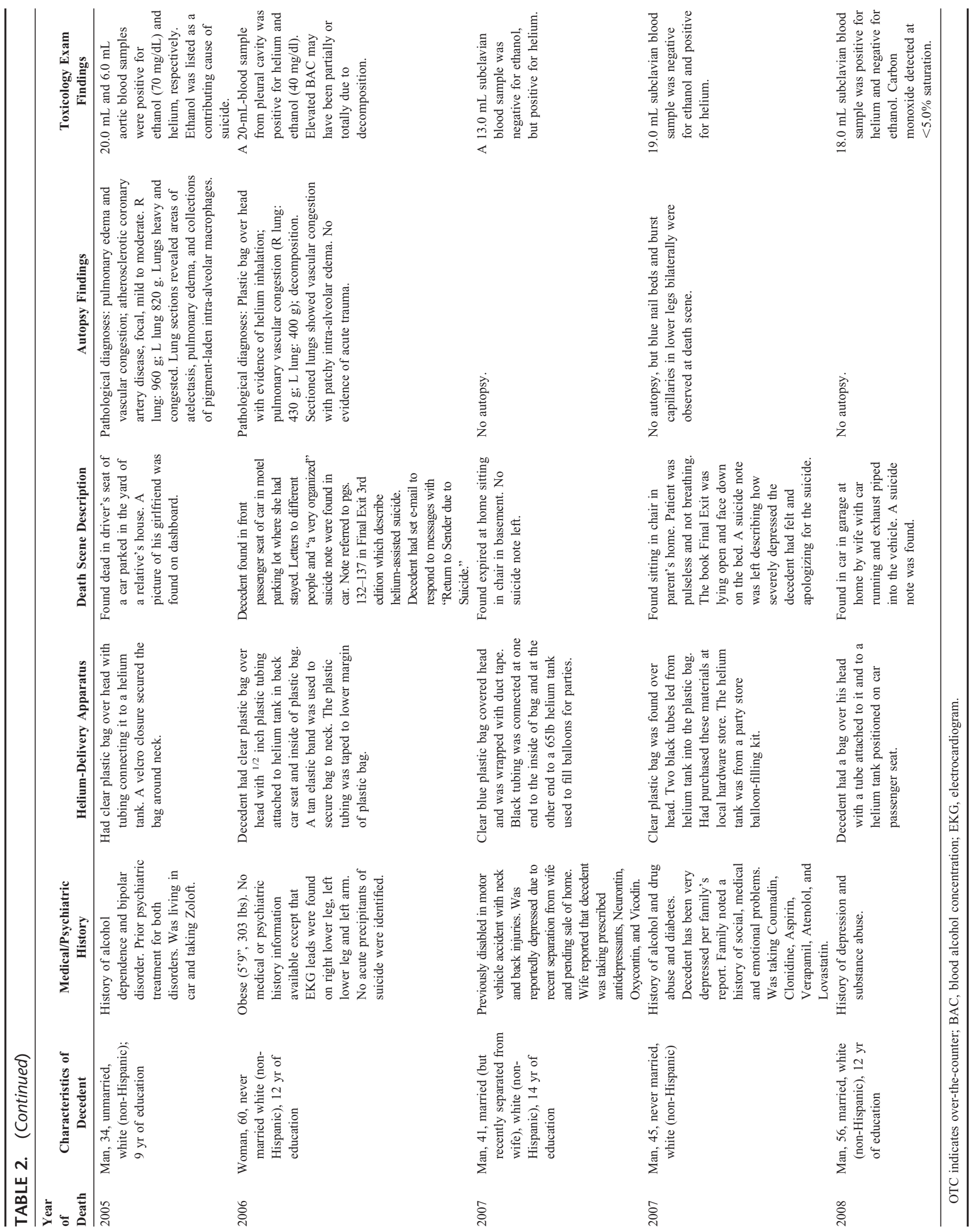


men who were relatively young ( $\mathrm{M}$ age, 41.1; $\mathrm{SD}$, 11.6; range, 21-60; median, 40.0). In 6 of 10 cases, decedents suffered from significant psychiatric dysfunction; in 3 of these 6 cases, psychiatric problems were present comorbidly with substance abuse. Medical histories identified chronic pain, disability, and chronic pain associated with injuries suffered in a motor vehicle accident, and diabetes (with probable coronary artery disease in 3 decedents). One decedent was found with electrocardiogram leads attached to her body, but autopsy and toxicological findings were negative for potential explanations for the death other than helium-assisted suicide. In none of the 10 cases were decedents suffering from terminal illness.

Helium delivery devices were consistent with those recommended in Final Exit (eg, use of T-valves, 2 helium tanks, Velcro and other neck fasteners), and all were associated with use of a plastic bag rather than mask. ${ }^{8}$ In 5 cases, a suicide note was found; in 2 cases, a will was left; in 1 case, insurance papers were left; and in 2 cases, right-to-die materials were found

Autopsies were performed in a majority of cases and typically revealed evidence of pulmonary vascular congestion and mild cerebral edema. Ethanol and diphenhydramine were considered contributing causes of death in 1 case each.

\section{DISCUSSION}

Despite reports identifying a plethora of prosuicide internet sites providing detailed instructions in methods of suicide including helium-assisted asphyxiation, ${ }^{18}$ media accounts of helium-assisted suicides, ${ }^{19-21}$ and the recent arrests of Final Exit Network members for allegedly assisting in asphyxial suicides involving helium, ${ }^{22}$ scientific investigations of such suicides are largely absent from the medical literature. This dearth of information is unfortunate given the tragic consequences of such acts and because it is possible that suicides by the helium method are underestimated and increasingly common for reasons described later in the text.

The methods by which helium-assisted suicides are carried out have been carefully detailed and widely publicized and the approach is promoted as simple, painless, and quick. ${ }^{8}$ Materials needed for helium-assisted suicides are easily obtained and inexpensive. One well-known internet vendor currently sells disposable helium tanks for less than $\$ 50$, and reports that customers who bought helium tanks also often bought the book Final Exit. ${ }^{23}$ Unless there is a high index of suspicion for helium involvement in a death, the death may be erroneously attributed to natural causes or underlying illness because standard toxicological assays are unlikely to detect helium and autopsy findings are generally nonspecific. ${ }^{14,16}$ Standard toxicological assays using GC/MS employ helium as the carrier gas and therefore cannot detect helium unless another gas (eg, nitrogen) is substituted for the helium. Auwaeter et $\mathrm{al}^{14}$ and Gallagher et $\mathrm{al}^{13}$ developed useful methods of collecting, preserving, and analyzing gas samples taken from decedents' for qualitative detection of helium. In all North Carolina cases, helium-delivery devices were found at the death scene, and toxicological testing was conducted in 9 of 10 cases. However, it is possible that an unknown number of such suicides went undetected, if and when heliumdelivery devices and plastic bags were removed from the death scene prior to investigation. The author of Final Exit states that a person may choose to leave right-to-die materials to be found to make an ethical statement that they are committing "rational suicide" or, conversely, make plans to have the helium delivery apparatus and plastic bag removed following their death if they prefer to keep the suicidal nature of the death concealed. ${ }^{8}$ Toxicological testing for helium has been conducted at the NCOCME in suspected cases since 2003 by a novel testing procedure using a dual cell thermal conductivity detector. ${ }^{24}$
Of particular concern, are recent national reports of notable increases in the prevalence of suicide due to suffocation (a category that includes deaths by plastic bag asphyxiation with or without helium assistance as well as hanging and strangulation) since the 1990 s and especially since $2000 .^{25-27}$ Such increases have been observed in respondents of widely varying ages, including adolescents, and both genders. Observers have noted that the reasons for these increases are poorly understood, that declining rates of suicide observed in the 1990s have been largely reversed, and that recent increases in suicides due to suffocation account for most of the recent overall increases in rates of suicide. ${ }^{28}$ It is possible that greater awareness of the plastic bag asphyxiation method and the enhanced lethality of the method when used with helium may account for the significant increases in suicides due to suffocation reported since 2000 .

Given the national growth in adolescent, young adult, and adult suffocation suicides since $2000,{ }^{25,26}$ and relatively young age, psychiatric dysfunction, and absence of terminal illness characteristic of many identified cases, it is possible that many persons committing suicide by the helium method are neither hopelessly nor terminally ill, but rather psychiatrically disordered. Although the author of Final Exit cautions readers to be certain they are hopelessly ill, and not just depressed and to talk to their doctor, ${ }^{8}$ depressive illness and substance dependency often impair the very capacities required to make these assessments and undertake these actions.

Prospective studies are needed to better understand the prevalence, incidence, predictors, and characteristics of asphyxial suicides due to helium inhalation. It is important to learn more about decedents' medical and psychiatric histories and the circumstances in which depressed and/or suicidal persons encounter descriptions of the helium method (eg, internet demonstrations of the process). At present, professionals working with persons at risk for suicide should routinely assess whether patients have read or viewed instructional materials describing specific methods of suicide such as helium-assisted plastic bag asphyxiation. Inquiries of this nature do not increase subsequent risk for suicide and can provide critically important information to guide appropriate preventative actions where indicated. ${ }^{29,30}$ Medical examiners should also increase their index of suspicion for suicides by asphyxiation associated with helium inhalation. Medical ethicists and the general public may also want to carefully weigh the unintended adverse consequences of widely disseminated suicide methods likely to appeal to some depressed persons (irrespective of their physical health status or age) against the putative benefits associated with making these methods more widely known and available.

\section{ACKNOWLEDGMENTS}

The authors thank P. Barnes, Administrative Services Manager, and other staff of the North Carolina Office of the Chief Medical Examiner for their assistance.

\section{REFERENCES}

1. Humphry D. Final Exit: The Practicalities of Self-Deliverance and Assisted Suicide for the Dying. New York, NY: Delta; 1991.

2. Côté R. In Search of Gentle Death: A Brief History of the NuTech Group. Mt Pleasant, SC: Corinthian; 2008.

3. The most memorable books of the last 25 years: 25 books that leave a legacy. April 9, 2007. Available at: http://www.usatoday.com/life/top25-books.htm. Accessed November 6, 2009.

4. Sacks MH, Kemperman I. Final exit as a manual for suicide in depressed patients. Am J Psychiatry. 1992;149:842.

5. Marzuk PM, Tardiff K, Hirsch CS, et al. Increase in suicide by asphyxiation in New York City after the publication of final exit. $N$ Engl J Med. 1993;329:1508-1510

6. Marzuk PM, Tardiff K, Leon AC. Increase in fatal suicidal poisonings and 
suffocations in the year final exit was published. Am J Psychiatry. 1994;151: 1813-1814.

7. Humphry D. Supplement to Final Exit: The Latest How-to and Why of Euthanasia/Hastened Death. Junction City, OR: Norris Lane and ERGO; 2000.

8. Humphry D. Final Exit: The Practicalities of Self-Deliverance and Assisted Suicide for the Dying. 3rd ed. New York, NY: Delta; 2002.

9. Humphry D. Final Exit: The Practicalities of Self-deliverance and Assisted Suicide for the Dying. 3rd ed. Addendum. Junction City, OR: ERGO; 2009.

10. Humphry D. Final Exit on DVD: The Art of Self-deliverance From a Terminal Illness. Junction City, OR: ERGO; 2006. ISBN: 978-0-9768283-0-3.

11. Ogden RD, Wooten RH. Asphyxial suicide with helium and a plastic bag. Am J Forensic Med Pathol. 2002;23:234-237.

12. Gilson T, Parks BO, Porterfield CM. Suicide with inert gases: Addendum to Final Exit. Am J Forensic Med Pathol. 2003;24:306-308.

13. Gallagher KE, Smith DM, Mellen PF. Suicidal asphyxiation by using pure helium gas: case report, review, and discussion of the influence of the internet. Am J Forensic Med Pathol. 2003;24:361-363.

14. Auwaeter V, Perdekamp MG, Kempf J, et al. Toxicological analysis after asphyxial suicide with helium and a plastic bag. Forensic Sci Int. 2007;170: $139-141$.

15. Grassberger M, Krauskopf A. Suicidal asphyxiation with helium: Report of three cases. Wien Klin Wochenschr. 2007;119:323-325.

16. Schon CA, Ketterer T. Asphyxial suicide by inhalation of helium inside a plastic bag. Am J Forensic Med Pathol. 2007;28:364-367.

17. Barnung SK, Feddersen C. Suicide by inhaling helium inside a plastic bag[In Danish]. Ugeskr Laeger. 2004;166:3506-3507.

18. Recupero PR, Harms SE, Noble JM. Googling suicide: suicide information on the internet. J Clin Psychiatry. 2008;69:878-888.

19. Tyson AS. Military investigates West Point suicides. Washington Post. January 30, 2009
20. Ward D. Helium in an "exit bag" new choice for suicide: at least 19 people in B.C. have used method since 1999. Vancouver Sun. December 8, 2007.

21. Lam A. Asian Americans' rising suicide rates - three students take their lives. New American Media. August 13, 2009.

22. Bowers P. Final exit: compassion or assisted suicide? Time. March 2, 2009.

23. Disposable helium tank for sale at Amazon.com. Available at: http://wwsw amazon.com/BuyCostumes-Disposable-Helium-Tank/dp/B000WR8QQG/ ref=pd_sbs_hpc_3. Accessed November 6, 2009.

24. Poklis JL, Garside D, Gaffney-Kraft M, et al. A qualitative method for the detection of helium in postmortem blood and tissues. In: Proceedings from the Society of Forensic Toxicologists; October 17-21, 2005; Nashville, TN.

25. Lubell KM, Swahn MH, Crosby AE, et al. Methods of suicide among persons aged 10-19 years-United States, 1992-2001. MMWR Morb Mortal Weekly Rep. 2004;53:471-474.

26. Lubell KM, Kegler SR, Crosby AE, et al. Suicide trends among youths and young adults aged 10-24 years-United States, 1990-2004. MMWR Morb Mortal Weekly Rep. 2007;56:905-908.

27. Hu G, Wilcox HC, Wissow L, et al. Midlife-suicide: an increasing problem in U. S.Whites, 1999-2005. Am J Prev Med. 2008;35:589-593.

28. Barber C. Trends and rates in methods of suicide: United States, 1985-2004. Harvard Injury Control Research Center (cbarber@hsph.harvard.edu). Report based on data from the Web-based Injury Statistics and Reporting System (WISQARS), National Center for Injury Prevention and Control, Centers for Disease Control and Prevention, U.S. Vital Statistics. Available at: http:// www.cdc.gov/ncipc/wisqars.

29. Gould MS, Marrocco FA, Kleinman MS, et al. Evaluating iatrogenic risk of youth suicide screening programs: a randomized controlled trial. JAMA. 2005;293:1635-1643.

30. American Psychiatric Association.Practice guidelines for the assessment and treatment of patients with suicidal behaviors. In: Practice Guidelines for the Treatment of Psychiatric Disorders Compendium. 2nd ed. Arlington, VA: American Psychiatric Association; 2004:835-1027. 